Pirineos. Revista de Ecología de Montaña

vol. 176

Jaca, Enero-Diciembre, 2021, e065

ISSN-1: 0373-2568

https://doi.org/10.3989/pirineos.2021.176003

\title{
MEDIDAS DE MINIMIZACIÓN DE IMPACTOS MEDIOAMBIENTALES EN LA ORGANIZACIÓN Y CELEBRACIÓN DE CARRERAS DE MONTAÑA ¿QUÉ OPINAN LOS PARTICIPANTES?
}

\section{Measures to minimize the environmental impact of trail running races. What do the participants think?}

\author{
Estela Inés Farías-Torbidoni*1-2 ${ }^{\text {, Víctor Dorado Martínez }}{ }^{1-2}$, María Alejandra Martínez ${ }^{1}$ \\ ${ }^{1}$ Institut Nacional d'Educació Física de Catalunya (INEFC), Universidad de Lleida, Partida la Caparella s/n, Lleida, España. \\ 2 Grup d'Investigació Social i Educativa de l'Activitat Física i l'Esport
}

\begin{abstract}
Identificador ORCID de los autores y e.mail Estela Inés Farías Torbidoni: https://orcid.org/0000-0002-7541-8140. E-mail: efarias@gencat.cat Víctor Dorado: https://orcid.org/0000-0002-7253-8083. E-mail: vdorado@gencat.cat María Alejandra Martínez: https://orcid.org/0000-0003-4423-0046 E-mail: mamartinez@inefc.es *Autor corresponsal
\end{abstract}

Recibido: 14-02-2021. Aceptado: 15-04-2021. Fecha de publicación on-line: 18/06/2021

\begin{abstract}
Citation/ Cómo citar este artículo: Farías-Torbidoni, E.I., Dorado Martínez, V., Martínez, M.A. (2021). Medidas de minimización de impactos medioambientales en la organización y celebración de carreras de montaña ¿Qué opinan los participantes? Pirineos, 176, e065. https://doi.org/10.3989/pirineos.2021.175003
\end{abstract}

RESUMEN: Diferentes tipos de iniciativas han intentado aunar esfuerzos en la búsqueda de alternativas que contribuyan en la minimización de impactos medioambientales en la organización de carreras por montaña. Un buen ejemplo de estas iniciativas ha sido la reciente publicación de dos guías de buenas prácticas elaboradas a partir del trabajo de dos grupos de expertos. Pero proponer, no es lo mismo que aceptar. El camino hacia el consenso, en el caso de ser necesario, no es instantáneo, sino que es la suma de un intenso diálogo entre las partes implicadas, y de un ida y vuelta de aciertos y errores que solo pueden ser detectados con un adecuado seguimiento. Tomando como caso de estudio la Salomon Ultra Pirineu, dos fueron los objetivos perseguidos con la realización de este estudio: 1) avanzar en el conocimiento del perfil genérico de los practicantes de esta disciplina deportiva y 2) evaluar el grado de aceptación de las recomendaciones propuestas en ambas guías por parte de los participantes de este evento. Entre los principales resultados obtenidos destacan, por un lado, la presencia de un elevado grado de aceptación de la mayor parte de las recomendaciones propuestas en 


\title{
$2 \cdot$ AUTORES
}

ambas guías (70\%) y por otro, la constatación de la existencia de tres recomendaciones especialmente críticas, si al bajo grado de aceptación por parte de los participantes y alto grado de afectación hacia el medio natural hacemos referencia: desarrollo de las pruebas durante el horario nocturno y meses de primavera.

PALABRAS CLAVE: Eventos deportivos; Trail Running; impactos medioambientales; conciencia medioambiental; grado de aceptación.

\begin{abstract}
Different types of initiatives have tried to join forces in the search for alternatives that contribute to the minimization of environmental impacts in the organization of mountain races. A good example of these initiatives has been the development and recent publication of two good practice guides elaborated by two groups of experts. But proposing is not the same as accepting. The path to consensus, if necessary, is not instantaneous, but is the sum of an intense dialogue between the parties and a back and forth of successes and mistakes that can only be detected with adequate monitoring. Taking the Salomon Ultra Pirineu as a case study, two were the objectives pursued: 1) advance in the knowledge of the generic profile of the practitioners of this sport discipline and 2) assess the degree of acceptance of the proposed recommendations in both guides by the participants of this event. The main results obtained include, on one hand, the presence of a high degree of acceptance of most of the recommendations proposed in both guides (70\%) and, on the other, the verification of the existence of three especially critical recommendations if at the low degree of acceptance by the participants and high degree of affectation towards the natural environment we refer to: development of the tests during the night hours and spring months.
\end{abstract}

KEY WORDS: Outdoor sport events; Trail Running; environmental impacts; environmental awareness; level of acceptance.

\section{Introducción}

\subsection{El auge de los eventos deportivos en el medio natural}

En la actualidad, existe un amplio consenso en cuanto al auge que ha experimentado la organización y participación de eventos deportivos en el medio natural, entre los cuales la disciplina de carreras por montaña no ha sido una excepción (FEDME, 2011; Bataller et al., 2014; Pucurull, 2014; Llopis \& Vilanova, 2015; EUROPARC-España, 2016; Farías-Torbidoni et al., 2017; Seguí \& Farías-Torbidoni, 2018). Así lo demuestran datos como los obtenidos por Pucurull (2014) o Seguí \& Farías-Torbidoni (2018) en torno al incremento de la oferta o la demanda de esta disciplina deportiva referida de forma indistinta como Carreras de montaña (especialidad deportiva de la Real Federación Española de Atletismo), Carreras por montaña (especialidad deportiva de la Federación Española de Deportes de Montaña y Escalada) o Trail Running (TR) (Babí et al., 2021). Citar, por ejemplo, los resultados obtenidos por Pucurull (2014) respecto al número de inscritos registrado en algunas de las 150 carreras de montaña más importantes y significativas de la Comunidad Autónoma de Cataluña durante el periodo 2011-2013, que soportan la existencia de un incremento acumulado en torno a un $33 \%(19 \%, 12 \%$ y $2 \%$, según se contemplen los años 2011, 2012 y 2013, respectivamente). O los datos obtenidos por Seguí \& Farías-Torbidoni (2018) en la sistematización del año de edición de las 1228 carreras o marchas por montaña ofertadas durante el año 2015 en
España, que permitieron corroborar, para idéntico periodo, la presencia de un incremento acumulado de la oferta de este tipo de eventos deportivos en torno al $40 \%$.

Al respecto, y a pesar de que son innegables los importantes beneficios que el incremento de la organización y participación de este tipo de eventos puede aportar en la sociedad, ya sea a nivel social (ej. incremento de los niveles de práctica deportiva, creación de sentido de comunidad), ambiental (ej. revalorización del medio natural) o económico (ej. estímulo del desarrollo local), entre otros (Babí, 2019; FEDME, 2011; Fredline, 2005; Parra et al., 2014), resulta oportuno no perder de vista los posibles impactos negativos que del incremento de la organización de este tipo de eventos se pueden derivar (Fraguas et al., 2008; Pernas et al., 2009), especialmente si al medio natural hacemos referencia (Marion et al., 2016; Newsome \& Hughes, 2017). De aquí, que la planificación y celebración de este tipo de eventos no debería ser realizada sin tener en cuenta la consecución de un doble objetivo que no siempre van de la mano: el de velar por minimizar los impactos negativos y maximizar los impactos positivos (Taks et al., 2014; Agha \& Taks, 2015; Hautbois et al., 2020).

Resulta oportuno destacar, en este punto, que de acuerdo a los datos publicados por Farías-Torbidoni et al., (2018), más del $82 \%$ de las carreras o marchas por montaña organizadas durante el año 2015 en España transcurrieron por algún Espacio Natural Protegido incluido en Red Natura 2000 y que más del $43 \%$ de las mismas fueron organizadas durante los meses de primavera. Meses, en este caso, considerados especialmente vulnerables en términos de conservación, si a la nidificación de aves, flo- 
ración de determinadas especies vegetales, etc. hacemos referencia (Meadema et al., 2020).

En términos generales, los impactos medioambientales que se pueden derivar de la celebración de este tipo de eventos no dependen de un solo factor. En este sentido, aunque sin ser específicos de la práctica de las carreras por montaña o TR, son numerosos los estudios que nos permiten identificar algunos de los principales impactos directos susceptibles de ser ocasionados durante la práctica de esta actividad (Leung \& Marion, 2000; Cole, 2008; Pickering, 2010; Newsome, 2014; Marion et al., 2016; Ng et al., 2018; Graefe et. al., 2019; Dujisin, 2020; Salesa \& Cerdà, 2020). Agrupados según los diferentes componentes estos pueden ser:

- Sobre la vegetación: Reducción de la altura y vigor de la vegetación, alteración de la composición de la misma, pérdida de cobertura vegetal, introducción de especies exóticas.

- Sobre el suelo: Pérdida de materia orgánica, desplazamiento del suelo, compactación del suelo, pérdida de la capacidad de infiltración, aumento de la conectividad hídrica.

- Sobre el agua: Incremento de la turbidez del agua, incremento de la entrada de nutrientes, introducción de organismos patógenos, alteración de la composición del agua.

- Sobre la fauna: Degradación o pérdida de hábitats, perturbación de la fauna, modificación del comportamiento habitual, desplazamiento a otros hábitats.

- Otros impactos: Perturbaciones por ruido, abandono de residuos, deposiciones de los participantes, marcaje y no retirada de señales, etc.

En este sentido resulta especialmente relevante tener en cuenta los estudios piloto llevados a cabo por Oñorbe (2014a, 2014b) y Benayas et al. (2015), que de alguna manera sentaron las bases, a través de una primera aproximación práctica a la problemática de compatibilización de usos, de lo que sería una de las primeras guías de buenas prácticas en la organización de carreras por montaña en espacios naturales protegidos.

\subsection{Guías o Códigos de buenas prácticas}

En este contexto, donde el incremento de la presión de este tipo de uso sobre el medio natural pone en evidencia la necesidad de una gestión activa sobre la organización de este tipo de eventos, surgen dos iniciativas encaminadas a encontrar un punto de encuentro entre los diferentes entes participantes (administraciones, entidades organizadoras, participantes) en el camino del consenso en la minimización de los impactos negativos y potenciación de los impactos positivos. Concretamente, la Guía de buenas prácticas para el desarrollo de carreras por montaña en espacios naturales protegidos, liderada en este caso por Europarc- España (2016) y el Codi de bones pràctiques en l'organització i celebració de curses i marxes per muntanya (Fundació Mon Rural, 2017). La primera, orientada más en la gestión de este tipo de eventos en el ámbito de los Espacios Naturales Protegidos y la segunda en el ámbito rural de una de las Comunidades Autónomas con mayor presencia de este tipo de eventos deportivos, como lo es la Comunidad Autónoma de Cataluña (Seguí \& Farías-Torbidoni, 2018).

En este sentido, el objetivo perseguido en ambas guías era proporcionar a las entidades organizadoras, a las administraciones locales o ambientales y a los participantes, mediante una serie de recomendaciones o limitaciones correctamente justificadas y consensuadas, un conjunto de criterios que guiaran el desarrollo de este tipo de eventos. En este punto cabe mencionar, que ambas guías fueron elaboradas a partir de la configuración de dos grupos de trabajo, con la participación, en el caso de la guía promovida por Europarc-España, de más de 40 representantes y expertos de las diferentes partes implicadas, y de una veintena en el caso del Codi de Bones Pràctiques.

En líneas generales, tres fueron los ámbitos de actuación considerados en el avance de una mayor compatibilización de usos vinculados a la organización de este tipo de eventos deportivos, ya sean estos en espacios naturales protegidos o no: 1) sobre las entidades organizadoras de la prueba, 2) sobre las administraciones responsables del espacio/territorio y 3) sobre los participantes.

Y es, en este último ámbito, el de los participantes, donde a veces tan buenas propuestas pueden quedar relegadas a solo buenas intenciones; ya que son los participantes, en última instancia, los que en definitiva deberán asumir las consecuencias de las correspondientes recomendaciones y/o restricciones. De aquí, que tener un mínimo de conocimiento acerca de las características principales de los practicantes de esta disciplina deportiva (incluidas sus motivaciones, preferencias y niveles de conocimiento o sensibilidad ambiental), como así también conocer la opinión o

Tabla 1. Resumen modalidades de competición Ultra Pirineu 2018. Table 1. Summary of the Ultra Pirineu 2018 competition modalities.

\begin{tabular}{|l|l|l|l|l|l|}
\hline Modalidad competición & Kilómetros & $\begin{array}{l}\text { Desnivel positivo } \\
(\mathbf{m})\end{array}$ & Plazas & $\begin{array}{l}\text { Puntos de avitua- } \\
\text { llamiento }\end{array}$ & Salida \\
\hline Ultra Pirineu & 110 & 6800 & 1000 & 11 & Bagà \\
\hline Maratón Pirineu & 45 & 2400 & 1000 & 5 & Bellver de Cerdanya \\
\hline Mitja Pirineu & 20 & 630 & 1000 & - & Bellver de Cerdanya \\
\hline Nit Pirineu & 5 & 860 & 200 & 1 & La Molina \\
\hline
\end{tabular}


nivel de aceptación de las recomendaciones o limitaciones propuestas por ambas guías, podría resultar de suma utilidad si se quiere avanzar hacia la implementación efectiva de este tipo de iniciativas. Esto es, viabilidad real y consolidación final de las recomendaciones o limitaciones sugeridas.

Por ello, y con la finalidad de aportar dichos datos, los objetivos de este artículo, tomando como caso de estudio la Ultra Trail Pirineu son: 1) identificar el perfil genérico de los participantes de este tipo de eventos y 2) analizar el nivel de aceptación por parte de estos en las recomendaciones de minimización de impactos ambientales promovidas por ambas guías.

\subsection{Características Ultra Trail Pirineu}

La Salomon Ultra Pirineu fue creada por Salomon (empresa de material deportivo) en el año 2009 sobre el trazado de una de las travesías más emblemáticas del Parc Natural del Cadí-Moixeró: Cavalls de Vents. Con el paso de los años el número de recorridos se fue ampliando y modificando hasta llegar a los cuatro recorridos incluidos en su décima edición: año 2018 (Tabla 1).

\section{Métodos}

\subsection{Participantes}

La muestra utilizada en este estudio estuvo compuesta por un total de 241 participantes de la Salomon Ultra Pirineu 2018. El procedimiento empleado para la selección de la muestra fue probabilístico, concretamente: aleatorio estratificado. El mismo fue implementado en base a la consideración del número total de participantes inscritos en cada uno de los diferentes recorridos del evento: 110 km, 45 km, 20 km y 5 km (Tabla 2).

\subsection{Procedimiento}

La encuesta fue realizada durante el mes de septiembre de 2018 durante dos días consecutivos: siendo los momentos de la implementación: (i) el día previo a la carrera, durante el horario de recogida de la bolsa del corredor, concretamente el viernes 29 de septiembre de 12:00 a las 19:00 horas (totalidad de corredores), y (ii) el día de la carrera, en el lugar de llegada (distancias de 20 y $45 \mathrm{~km}$ ).

La encuesta fue redactada en cuatro idiomas (catalán, castellano, inglés y francés) e implementada cara a cara con el empleo de tabletas. El programa utilizado fue el Kobotoolbox (Pham et al., 2019) y para la implementación se contó con la participación de un equipo de trabajo de 9 personas, conformado por los autores del trabajo y la colaboración de siete estudiantes de segundo curso de Ciencias de la Actividad Física y el Deporte que estaban cursando la asignatura de Sociología del Deporte.

\subsection{Instrumento}

Para la elaboración del modelo de encuesta utilizada se recurrió a la consulta de trabajos previos tales como los realizados por Baena \& Rebollo (2009), Llopis \& Llopis (2012) y Getz \& MacConnell (2014) entre otros, incluida una primera aproximación a la identificación del perfil genérico de corredores realizado por uno de los autores del presente estudio (Farías-Torbidoni et al., 2015). El diseño de la encuesta utilizada se basó en la contemplación de un total de 5 dimensiones claramente diferenciadas: 1) Sociotipo (edad, género, lugar de residencia habitual, etc.), 2) Historial deportivo TR y práctica deportiva (iniciación, experiencia, práctica deportiva, etc.), 3) Conocimientos y grado de sensibilidad ambiental (conocimiento de la categoría de protección del área en la cual se realiza el evento, impactos ambientales), 4) Motivaciones de práctica y de participación en el evento, y 5) Opiniones acerca las recomendaciones de reducción de impactos promovidas por ambas guías. En total, el modelo de encuesta quedó conformado por una total de 23 preguntas, la mayor parte de las cuales fueron cerradas o susceptibles de ser respondidas en consideración de diferentes escalas de Likert. La duración media de la realización de la encuesta fue de unos 8 minutos.

La elaboración del listado de recomendaciones se basó en la sistematización y unificación de la totalidad de las recomendaciones propuestas en la Guía de Buenas Prácticas promovida por Europarc-España y en el Codi de Bones pràcticas dinamizada por Fundación Mon Ru-

Tabla 2: Distribución de las encuestas realizadas en función de las distancias de recorrido.

Table 2: Distribution of the surveys carried out according to the distance covered.

\begin{tabular}{|l|l|l|}
\hline Recorridos evento & Núm. de inscritos (\%) & Número encuestas realizadas (\%) \\
\hline $5 \mathrm{~km}$ & $86(4 \%)$ & $10(4,1 \%)$ \\
\hline $20 \mathrm{~km}$ & $1071(50 \%)$ & $120(49,8 \%)$ \\
\hline $45 \mathrm{~km}$ & $321(15 \%)$ & $37(15,4 \%)$ \\
\hline $110 \mathrm{~km}$ & $600(28 \%)$ & $68(28,2 \%)$ \\
\hline Más de 1 distancia & $64(2,5 \%)$ & $6(2,5 \%)$ \\
\hline Total & $\mathbf{2 . 1 4 2}(\mathbf{1 0 0} \%)$ & $\mathbf{2 4 1}(\mathbf{1 0 0 \% )}$ \\
\hline
\end{tabular}


ral, tomando como referencia el estudio realizado por Benayas et al. (2015).

En este sentido vale la pena destacar que la información recogida en la dimensión 5 se basó en la realización de la siguiente pregunta: ¿Qué opinión le merece que desde las organizaciones de carreras de TR se apliquen las siguientes medidas de minimización de impactos medioambientales? y la contemplación de un total de 21 recomendaciones.

Finalmente, el modelo de encuesta fue revisado y valorado a dos niveles: grupos de expertos entre los cuales fueron incluidos miembros del grupo de trabajo de ambas guías y el Comité organizador de la prueba Salomon Ultra Pirineu.

\subsection{Análisis de datos}

El análisis de datos se llevó a cabo con el software SPSS (v. 15.0; SPSS Inc., IL) versión para Windows. Para la descripción de los mismos se emplearon los siguientes estadísticos: medias, desviación típica, intercuartiles y porcentajes.

\section{Resultados}

\subsection{Características sociodemográficas}

Entre las principales características sociodemográficas de los participantes (Tabla 3) destacan la identificación de un perfil medio de corredor/a de TR caracterizado por: ser de género masculino $(84,6 \%)$, tener edades comprendidas entre $26-45$ años (67,3\% - edad media de 35 años -), estar casado/a o en pareja (52,7\%), poseer estudios de nivel universitario $(39,4 \%)$, trabajar como empleado/a por cuenta ajena en un $45,2 \%$ y residir de forma habitual en España $(82,6 \%)$.

Tabla 3: Características principales de los participantes Salomon Ultra Trail Pirineu 2018.

Table 3: Main characteristics of the Salomon Ultra Trail Pirineu 2018 participants.

\begin{tabular}{|l|l|}
\hline Variables & $\begin{array}{l}\text { Porcentaje } \\
\mathrm{N}=241\end{array}$ \\
\hline Género & \\
\hline Masculino (\%) & 84,6 \\
\hline Femenino (\%) & 15,4 \\
\hline Grupos de edades & \\
\hline 16-25 años (\%) & 19,5 \\
\hline 26-35 años (\%) & 30,7 \\
\hline 36-45 años (\%) & 36,5 \\
\hline 46-55 años (\%) & 12,0 \\
\hline $56-65$ años (\%) & 0,9 \\
\hline
\end{tabular}

\begin{tabular}{|l|l|}
\hline Variables & $\begin{array}{l}\text { Porcentaje } \\
\mathrm{N}=241\end{array}$ \\
\hline Más de 65 años (\%) & 0,4 \\
\hline Edad (años). Media (SD). & $34,9(9,6)$ \\
\hline Situación familiar & \\
\hline Soltero/a (\%) & 41,1 \\
\hline Casado/a (\%) & 52,7 \\
\hline Otras situaciones (\%) & 6,1 \\
\hline Estudios más altos alcanzados & \\
\hline Estudios primarios (\%) & 1,3 \\
\hline Estudios secundarios (\%) & 9,1 \\
\hline Bachillerato - FP (\%) & 28,6 \\
\hline Estudios universitarios (\%) & 39,4 \\
\hline Estudios postgrado - máster (\%) & 18,7 \\
\hline Estudios postgrado - doctorado (\%) & 2,9 \\
\hline Dedicación laboral & \\
\hline Estudiante (\%) & 12,4 \\
\hline Parado/a (\%) & 2,9 \\
\hline Empleado/a público/a (\%) & 19,9 \\
\hline Empleado/a por cuenta ajena (\%) & 45,2 \\
\hline Empresario/a - autónomo/a (\%) & 17,4 \\
\hline Otras situaciones (\%) & 2,1 \\
\hline Lugar de residencia habitual & \\
\hline España (\%) & $8,2,6$ \\
\hline Portugal (\%) & 4,1 \\
\hline Andorra (\%) & 1,7 \\
\hline Ecuador (\%) & 1,7 \\
\hline Lituania (\%) & 1,7 \\
\hline Francia (\%) & 1,2 \\
\hline Argentina (\%) & \\
\hline Reino Unido (\%) & \\
\hline USA (\%) & \\
\hline Otros países (incluido un total de 10) $(\%)$ & \\
\hline & \\
\hline
\end{tabular}

Nota: Los datos continuos son expresados en media (SD) de acuerdo con su distribución.

\subsection{Experiencia y hábitos deportivos actuales}

Respecto a la experiencia previa en relación a la práctica del TR u otras disciplinas deportivas (Tabla 4), los resultados obtenidos demuestran la presencia de un reducido $10,8 \%$ que se ha iniciado en esta práctica deportiva durante los últimos 12 meses, situando el tiempo medio de práctica para el resto de los participantes en torno a los 6 años $(M=6,1)$. Así mismo, más de un 55\% declararon haberse iniciado en el TR a través de la práctica de carreras por asfalto, al tiempo que un $83,8 \%$ manifestó 
contar con experiencia previa en otras actividades deportivas. Destaca, en este sentido, el hecho de que la mayor parte de los corredores encuestados manifestó combinar la práctica del TR con otras actividades deportivas tales como la bicicleta de montaña o BTT $(28,6 \%)$, el ciclismo $(11,1 \%)$, el esquí $(7,1 \%)$ y la natación $(7,1 \%)$. Solo un $36,1 \%$ declaró estar federado, de los cuales solo un 27,8\% comentó estarlo en la Federación Española de Deportes de Montaña y Escalada (FEDME).

Tabla 4: Principales características historial deportivo participantes.

Table 4: Main characteristics of the participants'sports record.

\begin{tabular}{|l|l|}
\hline Variables & $\begin{array}{l}\text { Porcentaje } \\
\mathrm{N}=241\end{array}$ \\
\hline 5 años (\%) & 17,9 \\
\hline 6 a 10 años (\%) & 32,1 \\
\hline Más de 10 años (\%) & 5,0 \\
\hline Experiencia (años). Media (SD). & $6,1(5,3)$ \\
\hline Inicios en asfalto & \\
\hline Sí (\%) & 55,4 \\
\hline No (\%) & 44,6 \\
\hline $\begin{array}{l}\text { Experiencia deportiva previa en otras disci- } \\
\text { plinas }\end{array}$ & \\
\hline Sí (\%) & 83,8 \\
\hline No (\%) & 16,2 \\
\hline Actualmente la combina con otras disciplinas & \\
\hline Sí (\%) & 73,9 \\
\hline No (\%) & 26,1 \\
\hline Disciplinas deportivas más comunes & \\
\hline BTT (\%) & 28,6 \\
\hline Ciclismo (\%) & 11,1 \\
\hline
\end{tabular}

\begin{tabular}{|l|l|}
\hline Variables & $\begin{array}{l}\text { Porcentaje } \\
\mathrm{N}=241\end{array}$ \\
\hline Tiempo practicando TR & \\
\hline Menos de un año (\%) & 9,2 \\
\hline 1 año (\%) & 3,8 \\
\hline 2 años (\%) & 12,9 \\
\hline 3 años (\%) & 10,4 \\
\hline 4 años (\%) & 8,8 \\
\hline
\end{tabular}

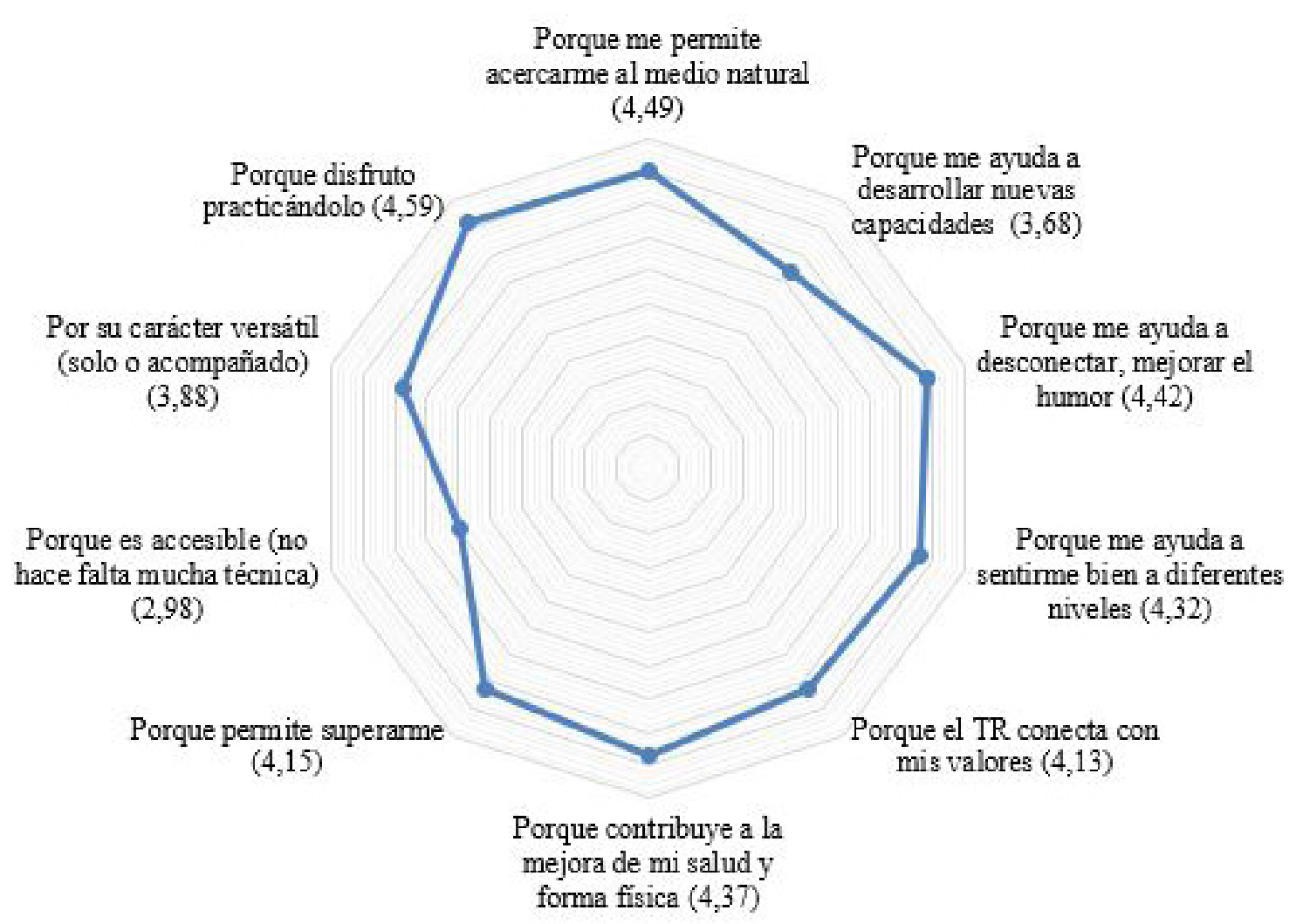

Figura 1: Motivaciones de práctica de TR participantes Salomon Ultra Pirineu 2018. Escala de Likert: 1 (no importante) - 5 (muy importante).

Figure 1: Practice motivations of TR participants Salomon Ultra Pirineu 2018. Likert scale: 1 (no important) - 5 (more important) 


\begin{tabular}{|l|l|}
\hline Variables & $\begin{array}{l}\text { Porcentaje } \\
\mathrm{N}=241\end{array}$ \\
\hline Esquí (\%) & 7,1 \\
\hline Natación (\%) & 7,1 \\
\hline Montañismo (\%) & 5,7 \\
\hline Fitness (\%) & 4,6 \\
\hline Escalada (\%) & 4,3 \\
\hline Otros (\%) & 31,5 \\
\hline No corresponde & 26,1 \\
\hline Esta federado & \\
\hline Sí (\%) & 36,1 \\
\hline No (\%) & 63,9 \\
\hline Federado en la FEDME & \\
\hline Sí (\%) & 27,8 \\
\hline No (\%) & 72,2 \\
\hline
\end{tabular}

Nota: Los datos continuos son expresados en media (SD) de acuerdo con su distribución.

\subsection{Motivaciones de práctica y de participación en el evento}

Dos fueron los bloques de motivaciones consultadas en este sentido: de práctica del TR y de participación en el evento. Tal y como se puede observar en las Figuras 1 y 2, las motivaciones de Disfrutar con la práctica del TR (con 4,59); Estar en contacto con el Medio Natural $(4,49)$; Relajarse y desconectar $(4,42)$ y Mejorar el estado de salud y forma física $(4,37)$, fueron las motivaciones más puntuadas en el caso de la práctica en sí misma, y de Pasar un buen rato $(4,42)$, Estar en forma $(4,12)$, Viajar o conocer nuevos lugares $(4,02)$ y Retarme a mí mismo $(3,82)$, las más puntuadas en el caso de la participación en el evento Salomon Ultra Pirineu.

\subsection{Grado de conocimiento y sensibilidad medioambiental}

En los resultados obtenidos en las cuatro preguntas incluidas en la dimensión de grado de conocimiento y sensibilidad medioambiental (Tabla 5) destacan el hecho

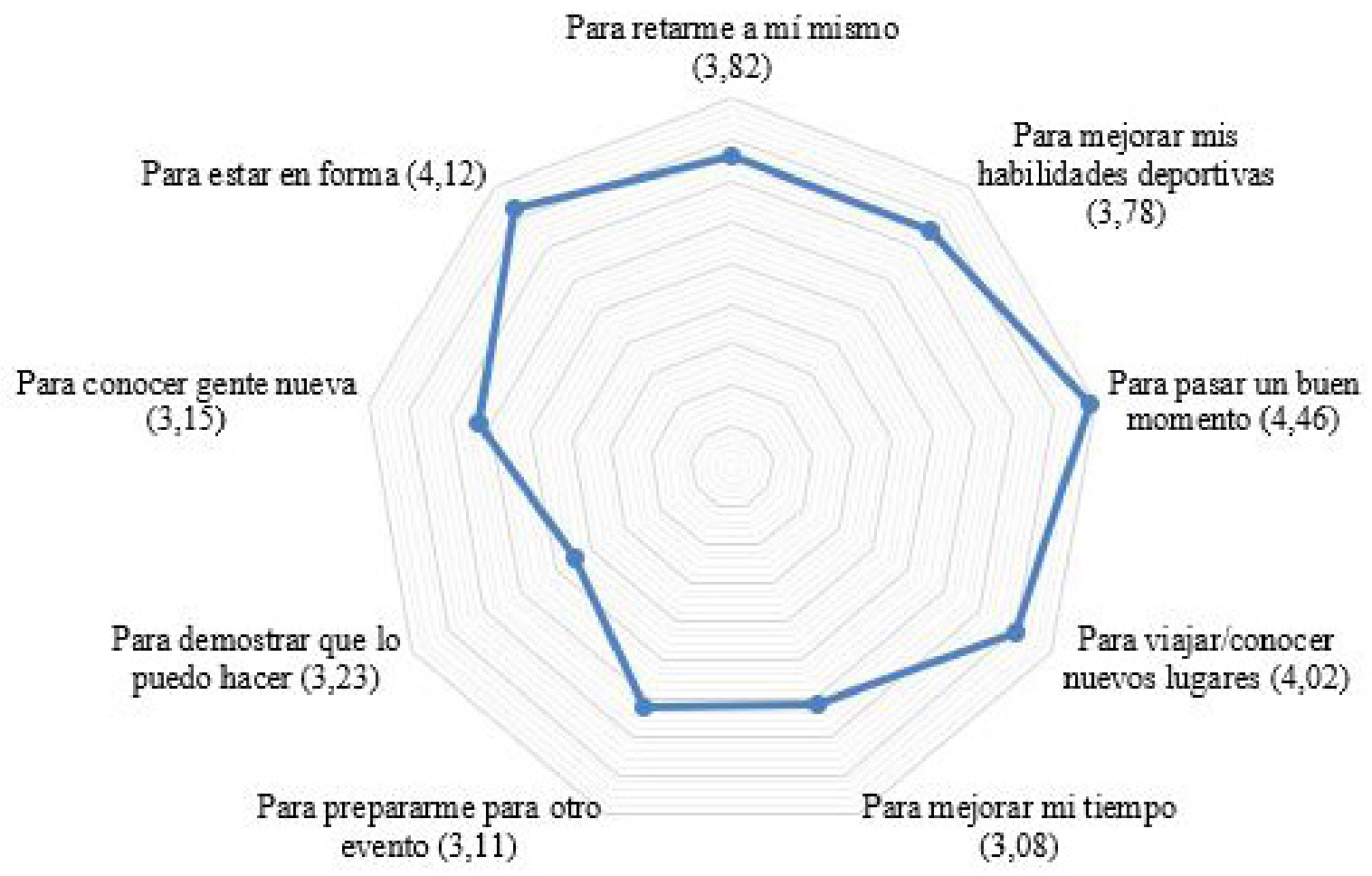

Figura 2: Motivaciones de participación en evento Salomon Ultra Pirineu 2018.

Figure 2: Motivaciones de participación en evento Salomon Ultra Pirineu 2018. 
de que la mayor parte de los encuestados no disponía de conocimientos previos acerca de la existencia de algunas de las guías o códigos de buenas prácticas $(52,1 \%)$, aunque sí eran conscientes de que el evento transcurría en un Espacio Natural Protegido (62,1\%). En este sentido, más de la mitad $(52,1 \%)$ de los corredores encuestados reconocieron que la práctica de esta actividad puede generar algún tipo de impacto medioambiental entre los cuales, un $41,3 \%$ fue capaz de identificar algún tipo de acción al respecto por parte de la entidad organizadora.

Tabla 5: Grado de conocimiento y sensibilidad medioambiental. Table 5: Degree of environmental knowledge and awareness.

\begin{tabular}{|l|l|}
\hline Variables & $\begin{array}{l}\text { Porcentaje } \\
\mathrm{N}=241\end{array}$ \\
\hline Conocimiento acerca de las guías & \\
\hline Sí (\%) & 14,9 \\
\hline No (\%) & 85,1 \\
\hline Conocimiento estatus de protección zona & \\
\hline No conocimiento (\%) & 29,6 \\
\hline No tiene nivel de protección (\%) & 8,3 \\
\hline Es un Espacios Natural Protegido (\%) & 62,1 \\
\hline Opinión sobre la generación de impactos & \\
\hline Sí (\%) & 52,1 \\
\hline No (\%) & 47,9 \\
\hline $\begin{array}{l}\text { Opinión acciones promovidas por la organiza- } \\
\text { ción para minimizar impactos }\end{array}$ & 47,9 \\
\hline No se generan impactos (\%) & 10,8 \\
\hline $\begin{array}{l}\text { No he observado ningún tipo de acción espe- } \\
\text { cífica (\%) }\end{array}$ & 41,3 \\
\hline $\begin{array}{l}\text { Si he observado acciones que contribuyen a la } \\
\text { reducción de impactos (\%) }\end{array}$ & \\
\hline
\end{tabular}

\subsection{Opinión sobre medidas de minimización de impacto}

Finalmente, en la Tabla 6 se pueden observar los resultados obtenidos en relación a las 21 recomendaciones propuestas por ambas guías incluidas en el modelo de encuesta.

Para una mejor interpretación de los resultados, las recomendaciones incluidas en la Tabla 6 han sido ordenadas de mayor a menor puntuación. Los números que se detallan al inicio de cada recomendación se corresponden al orden original establecido a partir de la contemplación de las diferentes fases presentes en la planificación y realización de cualquier tipo de evento (antes - durante - después).

Entendiendo que un mayor interés/viabilidad implica una mayor aceptación y un menor interés/viabilidad una menor aceptación, las puntuaciones obtenidas para el conjunto de las recomendaciones nos hablan de un nivel de aceptación bastante elevado, en tanto que 15 de las 21 recomendaciones $(71,4 \%)$ fueron valoradas dentro del rango muy interesante - interesantes o viables - muy viables (3-4). Al respecto, fueron las recomendaciones relacionadas con limitar o condicionar el horario (recomendación 5, $M=2,75)$, limitar el sobrevuelo de drones o helicópteros (recomendación 13: $M=2,79$ ), la época del año (recomendación 6: $M=2,95$ ), el trazado del recorrido (recomendación 7, $M=2,90$ ) y el uso libre de bastón (recomendaciones 14 y $15, M=2,86$ y 2,82 , respectivamente), las recomendaciones de minimización de impacto pero valoradas (puntuaciones inferiores a 3 ).

\section{Discusión}

El incremento de la presión sobre el medio natural como consecuencia del crecimiento en la organización de eventos deportivos en el medio natural requiere de una gestión activa que exige de un mínimo de información. Es aquí donde se centra la aportación de este estudio, en facilitar información que contribuya al conocimiento para una mayor compatibilización entre el aprovechamiento recreativo, deportivo y turístico del medio natural y su conservación.

Por un lado, y en relación con el perfil genérico, tan importantes a la hora de entender el porqué de algunas opiniones o reticencias en relación a la mayor o menor aceptación de determinados tipos de restricciones, destacan la identificación de un perfil sociodemográfico que no difiere en exceso de los resultados obtenidos en estudios previos como los llevados a cabo por Getz \& McConnell (2014) o Bataller et al. (2014), especialmente en lo que se refiere al género, edad y nivel de estudios alcanzados (léase: predominio de un perfil caracterizado por ser de género masculino, de mediana edad, con nivel de estudios de nivel medio-alto). Sin embargo, respecto al historial deportivo, a diferencia del estudio de Farías-Torbidoni et al. (2015), totalmente comparable en cuanto a la muestra y características de la prueba, los resultados obtenidos en este estudio señalan una tendencia creciente hacia la consolidación de un perfil de practicante más experimentado, con más experiencia no tan solo en la práctica, sino en el contacto con el medio natural, que bien podría sustentar, en parte, y de acuerdo a resultados obtenidos en estudios previos como los realizados por Heer et al. (2003), Huges \& Morrison-Saunders (2011), Farías-Torbidoni (2011), Barić et al. (2016) o Lin \& Lee (2020), el alto grado de conciencia ambiental identificado en el conjunto de los encuestados.

Válgase la mención, en este sentido, que más de la mitad de los participantes encuestados en este estudio respondieron de forma afirmativa a la pregunta relacionada con la posibilidad de que la práctica de su especialidad deportiva pudiese producir algún tipo de impacto medioambiental en el entorno en que se la realiza. En este caso, el 36\% más si tenemos en consideración los resultados obtenidos por Farías-Torbidoni \& Morera (2020) en una encuesta realizada a practicantes de diferentes actividades deportivas con un perfil más recreativo en el entorno del Parc Natural de la Serra de Collserola. Resultados, en este sentido, muy positivos en cuanto al entendimiento de la instauración de posibles nuevas regulaciones. 
Tabla 6: Opiniones medidas de minimización de impactos.

Table 6: Opinions on impact minimization measures.

\begin{tabular}{|c|c|c|}
\hline Recomendaciones & $\mathbf{M}$ & SD \\
\hline $\begin{array}{l}\text { 16. Penalizar o descalificar durante el evento el mal comportamiento o no cumplimiento de normas. Ejemplo, tirar } \\
\text { envases de geles, atajar, gritar en zonas sensibles señalizadas, etc. }\end{array}$ & 3,67 & 0,583 \\
\hline $\begin{array}{l}\text { 11. Evitar por parte de la organización el uso de pinturas, yeso o cal, o elementos no biodegradables para realizar el } \\
\text { marcaje del recorrido. }\end{array}$ & 3,56 & 0,631 \\
\hline $\begin{array}{l}\text { 8. Que la organización informe mediante e-mail a los participantes de las normas de conducta básicas a tener en } \\
\text { cuenta en la minimización de impacto. }\end{array}$ & 3,53 & 0,653 \\
\hline 12. Incluir cubos de separación de residuos en los avituallamientos instalados durante el recorrido. & 3,54 & 0,659 \\
\hline $\begin{array}{l}\text { 9. Hacer firmar a los participantes un compromiso de conducta que promueva el respeto del entorno natural o la } \\
\text { minimización de impactos. }\end{array}$ & 3,46 & 0,726 \\
\hline $\begin{array}{l}\text { 17. Poner a disposición de los corredores la posibilidad de denunciar a la organización cualquier conducta } \\
\text { medioambiental negligente de otros corredores. }\end{array}$ & 3,45 & 0,753 \\
\hline $\begin{array}{l}\text { 18. Identificar y dar a conocer a los acompañantes puntos óptimos de seguimiento de la carrera (horario aproxima- } \\
\text { do de paso incluido) que minimicen la afección ambiental en el entorno del recorrido. }\end{array}$ & 3,44 & 0,651 \\
\hline $\begin{array}{l}\text { 19. Ubicar carteles informativos en las zonas más sensibles a fin de que los corredores extremen las precauciones } \\
\text { (Ej. flora protegida para que no se pise, etc.). }\end{array}$ & 3,44 & 0,694 \\
\hline 3. Localizar el punto de salida y meta dentro de núcleos urbanos. & 3,31 & 0,683 \\
\hline $\begin{array}{l}\text { 10. Que la organización ponga a disposición de los participantes un vaso plegable reutilizable que se pudiera ir } \\
\text { utilizando a lo largo de todo el evento. }\end{array}$ & 3,27 & 0,887 \\
\hline 1. Habilitar transportes colectivos para los corredores y acompañantes. & 3,26 & 0,836 \\
\hline $\begin{array}{l}\text { 4. Promover la participación de los corredores en talleres de sensibilización medioambiental de forma previa o } \\
\text { posterior al evento. }\end{array}$ & 3,20 & 0,779 \\
\hline $\begin{array}{l}\text { 20. Hacer partícipes a los corredores en tareas de mantenimiento y restauración de los senderos por los que transcu- } \\
\text { rra la prueba y que hayan sido dañados por el desarrollo de ésta. }\end{array}$ & 3,18 & 0,818 \\
\hline $\begin{array}{l}\text { 21. Pagar de manera voluntaria un plus (entre } 1 \text { y } 2 € \text { ) en la inscripción del evento para que sea destinado a la res- } \\
\text { tauración o conservación del entorno que ha acogido la prueba. }\end{array}$ & 3,13 & 0,962 \\
\hline $\begin{array}{l}\text { 2. Premiar, de alguna forma por parte de la organización, la optimización en el uso de vehículos particulares (más } \\
\text { de } 3 \text { ocupantes) en el transporte al lugar del evento. }\end{array}$ & 3,10 & 0,832 \\
\hline $\begin{array}{l}\text { 6. Minimizar las competiciones durante la primavera para evitar afectar a ciertas especies de flora y fauna, espe- } \\
\text { cialmente aves. }\end{array}$ & 2,95 & 0,856 \\
\hline 7. Maximizar los trazados que se desarrollen por pistas forestales o red viaria rodada. & 2,90 & 0,980 \\
\hline 14. Limitar el uso de bastones en tramos en los que no se considere necesario (riesgo de erosión, humedad del terreno...). & 2,86 & 0,931 \\
\hline 15. Recomendar el uso de bastones sin punta metálica o con punta de goma. & 2,82 & 0,896 \\
\hline 13. Limitar el sobrevuelo de helicópteros, drones y otros aparatos. & 2,79 & 0,911 \\
\hline 5. Evitar al máximo el desarrollo del evento en horario nocturno para así minimizar la afección a fauna silves & 2,75 & 0,953 \\
\hline
\end{tabular}

Nota: Los datos continuos son expresados en media (SD) Desviación estándar. Puntuación Likert:1- nada interesante/viable, 2 poco interesante/viable, 3-interesante/viable y 4- muy interesante/viable Estos valores fueron calculados para una $N=241$.

Por otro lado, y a pesar de la diferencia en el enfoque $\mathrm{y}$ en el detalle de las medidas consideradas en estudios previos, los resultados obtenidos en la valoración del grado de interés/aceptación de las diferentes recomendaciones propuestas en ambas guías destacan por presentar una cierta coincidencia respecto a las recomendaciones menos aceptadas a los resultados obtenidos por Benayas et al. (2015): 1) bajo grado de aceptación de medidas relacionadas con la restricción de carreras en horario nocturno, 2) el condicionamiento del trazado principal del recorrido al uso de pistas y caminos preparados y 3) la prohibición de utilizar bastones durante el recorrido. Con la siguiente ex- cepción: menor grado de aceptación de la recomendación relacionada con la medida de restringir la realización de eventos durante los meses de primavera. Recomendación, en este caso, especialmente crítica si a la conservación del medio natural hacemos referencia y a las implicaciones prácticas y organizativas derivadas de este hecho: época del año con mejores condiciones para la práctica de esta actividad, temporada de competiciones, etc.

En este punto cabe destacar también la identificación de valores más elevados en aquellas recomendaciones ya ampliamente aceptadas en estudios previos que bien podrían ser el resultado del elevado nivel de conscien- 
cia o sensibilidad ambiental detectado en el perfil de los participantes y, por qué no suponerlo, del camino ya recorrido o en construcción de una nueva conciencia global enmarcada en la filosofía leave no trace cada vez más presente en el sistema deportivo mundial (Simon \& Alagona, 2009). Resultan especialmente relevantes, al respecto, algunas de las seis primeras recomendaciones más aceptadas, que lejos de no implicar o condicionar el comportamiento del corredor, ponen en evidencia su compromiso hacia y para la conservación que se debería potenciar a nivel de organización de este tipo de eventos. Concretamente: medida 16. Penalizar o descalificar durante el evento por el mal comportamiento o no cumplimiento de normas; 8 . Que la organización informe mediante e-mail a los participantes de las normas de conducta básicas a tener en cuenta en la minimización de impacto y medida 9. Hacer firmar a los participantes un compromiso de conducta que promueva el respeto del entorno natural o la minimización de impactos.

Finalmente, y concibiendo estas guías y este tipo de trabajos como unos primeros pasos en el avance hacia una gestión sostenible de este tipo de eventos, perfectamente y necesariamente transferibles a otras disciplinas deportivas con idiosincrasia o características similares, vale la pena destacar, la presencia de las siguientes limitaciones que se recomienda sean consideradas en la implementación de futuros estudios en esta línea de trabajo: 1) consideración de un solo evento deportivo, que podría no ser representativo al conjunto de los mismos si tenemos en cuenta la gran diversidad de organizadores y eventos existentes en esta y otras especialidades deportivas, 2) utilización del término interés - aceptación como un todo que se recomienda desglosar en trabajos futuros que esperamos se extiendan más allá de esta especialidad deportiva y 3 ) no inclusión en todo el proceso de la visión de otros actores como los promotores, población local o espectadores que sin lugar a dudas son parte implicada de todo el proceso y deberían ser considerados en el camino hacia una mayor sostenibilidad en la organización y celebración de este y otros tipos de eventos deportivos en el medio natural.

\section{Conclusiones}

En términos generales, los resultados obtenidos en el presente estudio sostienen la presencia de un perfil homogéneo caracterizado por ser hombres, de mediana edad, con nivel de estudios medios-alto, empleado por cuenta ajena y con una situación familiar que se podría considerar como estable. Se inició en el TR mediante la práctica de carreras por asfalto, que combina esta práctica con otras modalidades deportivas tales como la bicicleta todo terreno, el ciclismo, y el esquí, entre otras, que cuenta, en su gran mayoría con una experiencia practicando el TR superior a 4 años y que mayoritariamente no se encuentran federados. Destaca, en este sentido, la disposición de un buen nivel de conocimiento o sensibilidad medioambiental manifiesta no solo en cuestiones específicas rela- cionadas a la potencialidad de impacto de la práctica, sino que también en las motivaciones de práctica y de participación en este tipo de eventos.

En términos específicos, y en relación a la valoración de las 21 recomendaciones de minimización de impactos analizadas hay que destacar, por un lado, la identificación de un elevado grado de aceptación propuestas por ambas guías, manifestado en la obtención de más de un $70 \%$ de las mismas con valores superiores a 3 (interesantes - viables o muy interesantes - muy viables), dentro de una escala del 1 al 4 . Y por otro, la detección de un total de seis recomendaciones que requieren de un seguimiento especial por haber obtenido puntuaciones inferiores a 3 puntos. Concretamente y ordenadas de menor a mayor puntuación: Medida 5: Evitar al máximo el desarrollo del evento en horario nocturno para así minimizar la afección a fauna silvestre; medida 13: Limitar el sobrevuelo de helicópteros, drones y otros aparatos; medida 15: Recomendar el uso de bastones sin punta metálica o con punta de goma; medida 14: Limitar el uso de bastones en tramos en los que no se considere necesario (riesgo de erosión, humedad del terreno); medida 7: Maximizar los trazados que se desarrollen por pistas forestales o red viaria rodada; y medida 6: Minimizar las competiciones durante la primavera para evitar afectar a ciertas especies de flora y fauna, especialmente aves; que sin lugar a dudas podrían constituirse como un buen punto de partida experimental en el consenso de las partes implicadas.

A partir de las principales conclusiones obtenidas en este estudio dos podrían ser las ideas fuerza a considerar en el camino de una gestión más sostenible de este tipo de eventos: que el consenso no está tan lejos y que es necesario seguir trabajando en torno a la integración de las partes implicadas mediante la concienciación y el diálogo.

\section{Agradecimientos}

Organización Salomon Ultra Trail Pirineo y alumnos del Grado de Ciencias de la Actividad Física y Deporte del Institut Nacional d'Educació Física de Catalunya (INEFC). Universitat de Lleida: Olivia Bellaubí Palacios, Aleix Colell Sedano, Silvia García Blanco, Marina Herce Crespo, María Mas Canal, Joan Sancho Fiol, María Tejero Orduña.

\section{Referencias}

Agha, N. \& Taks, M., 2015. A theoretical comparison of the economic impact of large and small events. International Journal of Sport Finance, 10(3): 199-216. http://repository. usfca.edu/sm

Babí, J., 2019. La gestió de les curses a la muntanya a Catalunya. TDX (Tesis Dr. en Xarxa). Universitat de Barcelona, Barcelona.

Babí, J., Soler-Prat, S., Inglés-Yuba, E. \& Labrador-Roca, V., 2020. Historia y proceso de ordenación de las carreras en la montaña en España. RICYDE. Revista Internacional de Ciencias del Deporte, Volumen XVII: 140-159. https://doi. org/10.5232/ricyde2021.06403 
Baena, A. E., Rebollo Rico, S., 2009. Análisis del perfil sociodemográfico y competitivo del practicante de raids de aventura de ámbito nacional. Apuntes Educación Física y Deportes, 98: 68-77.

Barić, D., Petra, A, \& Macías-Bedoya, A., 2016. Segmenting protected area visitors by activities: A case study in Paklenica national Park. European Journal of Tourism Research, 13: $103-121$

Bataller, V., Marcén, C., Piedrafita, E. \& Arbonés, I., 2014. Comparación de los perfiles de corredores de carreras populares y corredores de carreras por montaña. XIII Congreso Internacional de la Asociación Española Investigación Sociológica Aplicada al Deporte, 10. https://www.zaragozadeporte.com/docs/Boletin/documento5133.pdf

Benayas, J., Oñorbe, M., Horcajo, L., 2015. Propuesta de medidas de gestión ambiental para la sostenibilidad de las carreras por montaña. Departamento de Ecología Universidad Autónoma de Madrid, 18. Doi: 10.13140/ RG.2.1.4131.5283

Cole, D., 2008. Ecological impacts of wilderness recreation and their management. In: Dawson, C.P., Hendee, J.C. (Eds.), Wilderness management: Stewardship and protection of resources and values. Fulcrum Press, Golden, CO, $395-438$.

Dujisin, P.R., 2020. Environmental impact generated by sports, recreation, and tourism activities in high mountain. Analysis of the mountain range of the Metropolitan Region of Santiago, Chile. Retos. Nuevas Tendencias en Educación Física, Deportiva y Recreación, 37: 62-69. https://doi.org/10.47197/ retos.v37i37.69036

EUROPARC-España, 2016. Guía de buenas prácticas para el desarrollo de carreras por montaña en espacios naturales protegidos, EUROPARC- España. Fundación Interuniversitaria Fernando González Bernáldez para los Espacios Naturales.

Farías-Torbiodni, E., 2011. Managing for recreational experience opportunities: The case of hikers in protected areas in Catalonia, Spain. Environmental Management, 47 (3): 482 496. https://doi.org/10.1007/s00267-010-9606-z

Farías-Torbidoni, E., Seguí, J. \& Inglés, E., 2015. Profiling participants of sporting events in the natural environment: trails running races. In: Plevnik, M., Retar, I., Pišot, R., Obid, A. (Eds.), Sustainable Development of Sports Tourism. Annales University Press, Koper: 185-194.

Farías-Torbidoni, E.I., Seguí, J., Mena, X., \& Sabaté, A., 2017. Eventos deportivos en espacios naturales protegidos. El caso de la red de Espacios Naturales de Protección Especial de Cataluña. Boletin Europarc España, 43: 29-31.

Farías-Torbidoni, E., Seguí, J., Ferrer, R., \& Dorado, V., 2018. Carreras de trail running y marchas por montaña en España. Número, evolución e incidencia sobre la Red Natura 2000. Pirineos, 173: 034. https://doi.org/10.3989/pirineos.2018.173001

Farías-Torbidoni, E.I, \& Morera, S., 2020. Estudi d'afluència, freqüentació i caracterització dels usuaris-visitants del Parc Natural de la Serra de Collserola. Consorci del Parc Natural de Collserola. Doi: 10.13140/RG.2.2.27890.12485

FEDME, 2011. Incidencia socioeconómica y ambiental de las carreras por montaña en el medio rural y natural de España. https://issuu.com/bibliotecafedme/docs/estudio_maquetado con portadas

Fraguas, A., Perero, E., Pérez, I, \& Queralt, J., 2008. Guía de medio ambiente y sostenibilidad, aplicados a los deportes no olímpicos 113. http://www.fundacioernestlluch.org/files/ Guia-Castellano.pdf

Fredline, E., 2005. Host and guest relations and sport tourism. Sport in Society, 8: 263-279. https://doi. org/10.1080/17430430500087328
Fundació Mon Rural, 2017. Codi de bones pràctiques en l'organització i celebració de curses i marxes per muntanya, $1^{\text {a }}$ edició. Ed. Entitat Autònoma del Diari Oficial i de Publicacions.

Getz, D. \& McConnell, A., 2014. Comparing Trail Runners and Mountain Bikers: Motivation, Involvement, Portfolios, and Event-Tourist Careers. Journal of Convation \& Event Tourism, 15: 69-100. https://doi.org/10.1080/15470148.2013.834807

Graefe, A., Mueller, J.T., Taff, B.D. \& Wimpey, J., 2019. A comprehensive method for evaluating the impacts of race events on protected lands. Society \& Natural Resources, 32: 11551170. https://doi.org/10.1080/08941920.2019.1583396

Hautbois, C., Djaballah, M. \& Desbordes, M., 2020. The social impact of participative sporting events: a cluster analysis of marathon participants based on perceived benefits. Sport in Society, 23(2): 335-353. https://doi.org/10.1080/17430437. 2019.1673371

Heer C., Rusterholz, H.P. \& Baur B., 2003. Forest perception and knowledge of hikers and mountain bikers in two different areas in northwestern Switzerland. Environmental Management, 31(6): 709-23. http://doi.org/10.1007/s00267003-3002-x

Hughes, M. \& Morrison-Saunders, A., 2011. Visitor attitues toward a modified natural attraction. Society \& Natural Resources, 16 (3): 191-203 https://doi. org/10.1080/08941920309160

Leung, Y.-F. \& Marion, J.L., 2000. Recreation Impacts and Management in Wilderness: A State-of-Knowledge Review The Field of Recreation Ecology. In: USDA Forest Service Proceedings: 23-48.

Lin, Y.H. \& Lee, T.H., 2020. How do recreation experiences affect visitors' environmentally responsible behavior? Evidence from recreationists visiting ancient trails in Taiwan. Journal of Sustainable Tourism, 28: 705-726. https://doi.org $/ 10.1080 / 09669582.2019 .1701679$

Llopis Goig, R., \& Llopis Goig, D., 2012. Assessment of the Thoracic Spine, Lumbar Spine and Pelvic Tilt in Elite and Master 30 Class Cyclists. Apunts. Educación Física y Deportes, 108: 9-16. https://dx.doi.org/10.5672/apunts.2014-0983. es.(2012/2).108.01

Llopis, R. \& Vilanova, A., 2015. Sociological Analysis of the Evolution and Characteristics of Running. In: J. Scheerder, K. Breedveld \& J. Borgers (Eds.), Running Across Europe. The Rise and Size of one of the Largest Sport Markets Palgrave. Macmillan: 220-240 pp., London.

Marion, J.L., Leung, Y.F., Eagleston, H. \& Burroughs, K., 2016. A review and synthesis of recreation ecology research findings on visitor impacts to wilderness and protected natural areas. Journal of Forestry, 114: 352-362. https://doi. org/10.5849/jof.15-498

Meadema, F., Marion, J.L., Arredondo, J. \& Wimpey, J., 2020. The influence of layout on Appalachian Trail soil loss, widening, and muddiness: Implications for sustainable trail design and management. Journal Environmental Management, 257: 109986. https://doi.org/10.1016/j.jenvman.2019.109986

Newsome, D., 2014. Appropriate policy development and research needs in response to adventure racing in protected areas. Biological Conservation, 171: 259-269. https://doi. org/10.1016/j.biocon.2014.01.008

Newsome, D. \& Hughes, M., 2017. The contemporary conservation reserve visitor phenomenon! Biodiversity and Conservation, 27: 521-529. https://doi.org/10.1007/s10531-017-1435-4

Ng, S.L., Leung, Y.F., Cheung, S.Y. \& Fang, W., 2018. Land degradation effects initiated by trail running events in an urban protected area of Hong Kong. Land Degradation \& Development, 29: 422-432. https://doi.org/10.1002/ ldr.2863 
Oñorbe, M., 2014a. Evaluación de impacto ambiental de carreras por montaña. Estudio piloto de la carrera por montaña del Demandafolk. Territorios Vivos. Madrid. https://www. miteco.gob.es/es/ceneam/recursos/pag-web/evaluacion-ambiental-carreras-montana.aspx

Oñorbe, M., 2014b. Guía orientativa para la evaluación ambiental de carreras por montaña que pueden afectar a espacios de Red Natura 2000 en Canarias. https://issuu.com/festucaelegans/docs/guia_ambiental_cxm_canarias_borrado

Parra, D., Calabuig, F., Añó, V. \& Ayora, D., 2014. El impacto de un evento deportivo mediano: percepción de los residentes de la comunidad de acogida. Retos. Nuevas Tendencias en Educación Física, Deporiva. y Recreación, 26: 88-93. https://doi.org/10.47197/retos.v0i26.34407

Pernas, J., Lucio, A., Pérez, P. \& Azcárate, J., 2009. Guía de buenas prácticas ambientales para eventos deportivos. http://femp.femp.es/files/566-1280-archivo/GUIA_VERDE_VERSION\%20DEFINITIVA.pdf

Pham, $\bar{P}$., Vinck, P., Kreutzer, T., Milner, J., Dorey, A., Musaraj, P., Olivier, L., Leszek, P., Coughlin, K., Aloo, S. \& de St. Aubin, M., 2019. KoBoToolbox | Data Collection Tools for Challenging Environments. Kobo Toolbox.

Pickering, C.M., 2010. Ten factors that affect the severity of environmental impacts of visitors in protected areas. Ambio, 39: 70-77. https://doi.org/10.1007/s13280-009-0007-6
Pucurull, M., 2014. 266 noves curses aquest any a Catalunya [WWW Document]. Calaix sastre del món del córrer. URL http://calaixsastremoncorrer.blogspot.com/2013/12/ noves-curses-aquest-any-2013-catalunya.html (accedido 9.1.20).

Salesa, D. \& Cerdà, A., 2020. Soil erosion on mountain trails as a consequence of recreational activities. A comprehensive review of the scientific literature. Journal of Environmental Management, 271: 110990. https://doi.org/10.1016/j.jenvman.2020.110990

Seguí, J. \& Farías-Torbidoni, E.I., 2018. El trail running (carreras de o por montaña) en España. Inicios, evolución y (actual) estado de la situación. Retos. Nuevas Tendencias en Educación Física, Deportiva. y Recreación, 33: 123-128. https://doi.org/10.47197/retos.v0i33.56462

Simon, G.L. \& Alagona, P., 2009. Beyond leave no trace. Ethics Place and Environment, 12(1): 17-34. https://doi. org/10.1080/13668790902753021

Taks, M., Green, B. C., Misener, L. \& Chalip, L., 2014. Evaluating sport development outcomes: The case of a medium-sized international sport event. European Sport Management Quarterly, 14(3): 213-237. https://doi.org/10.1080/ 16184742.2014 .882370 